\title{
Pazopanib maintenance after first-line etoposide and platinum chemotherapy in patients with extensive disease small-cell lung cancer: a multicentre, randomised, placebo- controlled Phase II study (KCSG-LU12-07)
}

Jong-Mu Sun ${ }^{1}$, Ki Hyeong Lee ${ }^{2}$, Bong-Seog Kim ${ }^{3}$, Hoon-Gu Kim ${ }^{4}$, Young Joo Min ${ }^{5}$, Seong Yoon Yi ${ }^{6}$, Hwan Jung Yun ${ }^{7}$, Sin-Ho Jung ${ }^{8}$, Se-Hoon Lee ${ }^{1}$, Jin Seok Ahn ${ }^{1}$, Keunchil Park ${ }^{1}$ and Myung-Ju Ahn ${ }^{\star}{ }^{1}$

${ }^{1}$ Division of Hematology-Oncology, Department of Medicine, Samsung Medical Center, Sungkyunkwan University School of Medicine, 81 Irwon-ro, Gangnam-gu, Seoul 06351, Korea; ${ }^{2}$ Division of Medical Oncology, Department of Medicine, Chungbuk National University Hospital, Chungbuk National University College of Medicine, Cheongju 28644, Korea; ${ }^{3}$ Division of HematoOncology, Department of Internal Medicine, Veterans Health Service Medical Center, Seoul 05368, Korea; ${ }^{4}$ Division of Hematology-Oncology, Department of Internal Medicine, Gyeongsang Institute of Health Sciences, Gyeongsang National University College of Medicine and Gyeongsang National University Changwon Hospital, Changwon 51472, Korea; ${ }^{5}$ Division of Hematology and Oncology, Department of Internal Medicine, Ulsan University Hospital, University of Ulsan College of Medicine, Ulsan 44033, Korea; 'Division of Hematology-Oncology, Department of Internal Medicine, Inje University Ilsan Paik Hospital, Goyang 10380, Korea; 'Division of Hematology-Oncology, Department of Medicine, Chungnam National University Hospital, Daejeon 35015, Korea and ${ }^{8}$ Department of Biostatistics and Bioinformatics, Duke University, Durham, NC 27710, USA

Background: We investigated whether pazopanib maintenance following first-line chemotherapy would improve survival in patients with extensive disease small-cell lung cancer (ED-SCLC).

Methods: This study is a randomised, placebo-controlled, phase II study that enroled ED-SCLC patients who had not progressed after four cycles of etoposide plus platinum therapy. Eligible patients were randomly assigned (1:1 ratio) to either placebo or pazopanib $800 \mathrm{mg}$ per day until progression or unacceptable toxicity. The primary end point was progression-free survival (PFS).

Results: 97 patients were enroled and randomly assigned; 2 patients did not receive study drugs. In total, 95 patients received maintenance therapy (pazopanib, $n=48$; placebo, $n=47$ ) and were included in the analyses. Grade 3 toxicities for pazopanib maintenance were thrombocytopenia (10.4\%, including one case with grade 4 toxicity), liver enzyme elevation (10.4\%), fatigue (6.3\%), and hypertension (6.3\%). Median PFS was 3.7 months for pazopanib maintenance and 1.8 months for placebo (hazard ratio $0.44,95 \%$ confidence interval: $0.29-0.69, P<0.0001)$.

Conclusions: Pazopanib maintenance significantly prolonged PFS in patients with ED-SCLC. Given the toxicity profiles, however, relevant biomarkers to select patients for benefit from pazopanib should be further investigated.

*Correspondence: Dr M-J Ahn; E-mail: silkahn@skku.edu

Received 21 August 2017; revised 24 November 2017; accepted 27 November 2017; published online 30 January 2018

(C) 2018 Cancer Research UK. All rights reserved 0007-0920/18 
Small-cell lung cancer (SCLC), which accounts for $\sim 15 \%$ of all lung cancers, is characterised by its rapid proliferation (Govindan et al, 2006). More than half of patients with SCLC are diagnosed with extensive disease (ED) and etoposide plus platinum is commonly used as first-line chemotherapy (Hanna et al, 2006). Despite dramatic response to first-line chemotherapy, most patients with ED-SCLC experience rapid relapse or progression during the off-therapy period (Hanna et al, 2006). Moreover, the clinical outcomes of subsequent chemotherapy for relapsed or progressive disease are unsatisfactory, resulting in a median overall survival of 9 to 10 months (Eckardt et al, 2007).

One approach to improve overall survival is maintenance therapy, which is administered in a state of non-progression after completion of first-line chemotherapy. Extensive disease-SCLC might theoretically be a good candidate for maintenance therapy, given that it is very short interval between completion of first-line chemotherapy and its subsequent disease progression (Schiller et al, 2001; Ready et al, 2015), and prolongation of this interval can eventually lead to improvement of overall survival. Four cycles of topotecan following first-line etoposide and cisplatin chemotherapy improved progression-free survival (PFS) but failed to improve overall survival or quality of life in ED-SCLC (Schiller et al, 2001).

Vascular endothelial growth factor (VEGF) is a proangiogenic factor that is important in pathologic angiogenesis in tumours. Small-cell lung cancer has a higher level of vascularisation than other tumours and is associated with high VEGF expression (Lucchi et al, 2002; Dowell et al, 2004). Therefore, antiangiogenic agents are expected to be good therapeutic agents for SCLC. A randomised phase II trial recently compared sunitinib maintenance therapy with placebo by analysing 85 patients with ED-SCLC (Ready et al, 2015). Sunitinib maintenance after four or six cycles of etoposide and platinum chemotherapy successfully met the primary endpoint, showing an increase in PFS from 2.1 months to 3.7 months $(P=0.02)$. Although it failed to show a significant benefit in terms of overall survival, the survival benefit by 2.1 months under the crossover design warranted a further large study to confirm the role of sunitinib maintenance in ED-SCLC.

Pazopanib is an oral angiogenesis inhibitor targeting VEGFR, PDGFR, and c-kit (Sonpavde and Hutson, 2007), and it has been approved in many countries for the treatment of patients with advanced renal cell carcinoma or advanced soft-tissue sarcoma (Sternberg et al, 2010; van der Graaf et al, 2012). Compared with the standard dose of sunitinib (50 mg per day for 4 of 6 weeks), pazopanib $800 \mathrm{mg}$ per day showed a comparable survival outcome with more tolerable toxicity profiles in patients with advanced renal cell carcinoma (Motzer et al, 2013). A single-arm phase II trial showed clinical activity of pazopanib for relapsed or refractory SCLC with a median PFS of 14.1 weeks (Gandhi et al, 2012).

Based on these data, the Korean Cancer Study Group-Lung 1207 trial evaluated pazopanib maintenance compared with placebo after standard therapy with four cycles of etoposide and platinum for untreated ED-SCLC.

\section{MATERIALS AND METHODS}

Study design and patients. This multicentre, phase II, placebocontrolled, double-blinded, randomised phase II trial was conducted at seven hospitals in South Korea. Patients were eligible if they were diagnosed with ED-SCLC and had no evidence of disease progression after four 21-day cycles of etoposide and platinum chemotherapy. The choice of platinum, either cisplatin or carboplatin, and the dose of the chemotherapy before enrollment depended on the physicians' discretion and did not affect enrollment as long as four cycles of chemotherapy were completed. Patients with asymptomatic brain metastases were eligible for inclusion. Patients should have Eastern Cooperative Oncology Group (ECOG) performance status of 0 to 2 and adequate organ function. If prophylactic cranial irradiation (PCI) was not planned, informed consent was required to be written between 21 and 42 days after day 1 of the fourth cycle of etoposide and platinum chemotherapy. If PCI was planned or already performe, d enrolment was required after completion of PCI and the allowed enrolment period was increased by 2 weeks.

The protocol was approved by the ethics review boards of each participating institution and all patients provided informed consent for enrolment in the trial. This study was sponsored by Novartis and registered in ClinicalTrials.gov (NCT0179784).

Randomisation. The enroled patients were stratified by ECOG performance status $(0,1$ vs 2$)$ and history of PCI (PCI vs no PCI). The stratified patients were randomly assigned in a $1: 1$ ratio to either the pazopanib or placebo arm. Randomisation was performed using a random number table generated for a stratified random permuted block design. The best response status of firstline chemotherapy did not affect the randomisation process.

Treatment procedures. Pazopanib $800 \mathrm{mg}$ or placebo was administered orally once daily every 28 days until disease progression, death, or unacceptable toxicity. To evaluate adverse events, patients were followed every 2 weeks for 2 months and every 4 weeks thereafter. Dose modification or interruption was performed according to the pre-planned guideline described in the study protocol. For evaluation of response or progression, computed tomography for chest or abdomen/pelvis was performed every two cycles. At the time of disease progression, patients received standard therapy at the physicians' discretion and crossover was not allowed.

Statistical analysis. The primary endpoint of this study was PFS, defined as the time from randomistion to disease progression or death from any cause. Secondary endpoints included overall survival and safety.

Sample-size calculation was based on the following assumption: the rate of 6-month PFS for the placebo arm was expected to be $20 \%$ based on historical data and we will be highly interested in pazopanib maintenance if the 6-month PFS is as high as $40 \%$. This study was expected to be able to randomise approximately five patients per month. Assuming 6 months of additional follow-up after completion of patient accrual and an exponential PFS model, 95 patients (about 48 patients per each arm) are required with $90 \%$ power to detect a difference of PFS by the two-sample log-rank test with one-sided $\alpha$ of $10 \%$. The final analysis was planned when 92 events (progression or death) were observed.

Statistical analysis was based on modified intent-to-treat principle including all patients who were eligible and did not withdraw consent prior to receiving treatment. The PFS and overall survival were analysed by Kaplan-Meier curves and compared by the log-rank test and Cox's regression method. The objective response was evaluated by RECIST 1.1 (Eisenhauer et al, 2009). Toxicity and its severity were evaluated according to the common terminology criteria for adverse events (version 4.0).

Each binary outcome was compared between the two arms using Fisher's exact test. The significance of statistical tests was evaluated based on a two-sided $\alpha$-value of $5 \%$ for the primary and other endpoints. Statistical analyses were performed using SAS version 9.4 (SAS Institute, Cary, NC, USA).

\section{RESULTS}

Patient characteristics. We screened 101 patients for eligibility from June 2013 to July 2016 and 97 patients were randomly allocated to either the pazopanib arm $(n=50)$ or the placebo arm 
$(n=47)$. Two patients in the pazopanib arm withdrew consent before receiving the study drug and were excluded from the modified intention-to-treatment analysis according to the study protocol (Figure 1). All baseline characteristics were well balanced between the two groups (Table 1). Approximately $90 \%$ of all participants were male or smokers. All participants had ECOG 0 or 1. Sixty-four percent of patients received etoposide and cisplatin and the others received etoposide and carboplatin as first-line chemotherapy before enrolment; $95.8 \%$ demonstrated partial response to first-line chemotherapy.

Adverse events and dose modification. In the pazopanib arm, common toxicities of any grade were thrombocytopenia (56.3\%), liver enzyme elevation (45.8\%), anorexia (41.7\%), proteinuria (33.3\%), skin lesions (33.3\%), diarrhoea (31.2\%), fatigue $(29.2 \%)$, stomatitis (25.0\%), thyroiditis (22.9\%), dyspepsia (20.8\%), and hypertension (16.7\%) (Table 2). Grade 3 toxicities with incidence $>5 \%$ were thrombocytopenia $(10.4 \%$, including one case with grade 4 toxicity), liver enzyme elevation (10.4\%), fatigue (6.3\%), and hypertension (6.3\%). There was no fatal event related to pazopanib maintenance.

Twenty-five patients (52.1\%) in the pazopanib arm experienced interruption of pazopanib administration due to adverse events and four of them discontinued treatment permanently due to unacceptable toxicities. The main reasons for permanent discontinuation included grade 2 proteinuria, prolonged grade 2 anorexia with grade 2 fatigue, grade 3 stomatitis with grade 2 diarrhoea and anorexia, and patient's refusal due to grade 1 anorexia with grade 1 hypertension. In the placebo arm, one patient interrupted the study after reporting grade 2 anorexia with grade 2 fatigue and was taken off the study.

In total, 25 patients $(52.1 \%)$ in the pazopanib arm required dose reduction due to adverse events: 16 patients $(33.3 \%)$ required dose reduction by 1 level (from 800 to $600 \mathrm{mg}$ per day) and 9 patients (18.8\%) required dose reduction by 2 levels to $400 \mathrm{mg}$ per day. In total, $63 \%$ of episodes of dose reduction occurred within 6 weeks after starting pazopanib maintenance treatment, whereas only one patient in the placebo arm required dose reduction.

Efficacy. On the day of data cut off (17 April 2017), 92 patients experienced disease progression or death. At a median follow-up of
30.1 months, the median PFS was 3.7 months (95\% confidence interval (CI): 3.5-3.8 months) for the pazopanib arm vs 1.8 months (95\% CI: 1.8-1.9 months) for the placebo arm (hazard ratio (HR) $0.44,95 \%$ CI: $0.29-0.69, P<0.0001$ ) (Figure 2). The number of patients with long duration of PFS ( $>6$ months) was $9(18.8 \%)$ in the pazopanib arm and $2(4.3 \%)$ in the placebo arm $(P=0.027)$. Three patients achieved additional partial remission during pazopanib maintenance therapy, whereas there was no partial or complete remission in the placebo arm.

The median overall survival for the pazopanib and placebo arms was 10.6 months (95\% CI: 8.4-12.7 months) and 12.9 months

\begin{tabular}{|c|c|c|c|}
\hline & $\begin{array}{c}\text { Pazopanib } \\
\operatorname{arm}(n=48, \\
100 \%)\end{array}$ & $\begin{array}{c}\text { Placebo } \\
\operatorname{arm}(n=47, \\
100 \%)\end{array}$ & $P$ \\
\hline Median age, years (range) & $66.5(57-79)$ & $67(50-83)$ & \\
\hline $\begin{array}{l}\text { Gender } \\
\text { Male } \\
\text { Female }\end{array}$ & $\begin{array}{c}40 \text { (83.3\%) } \\
8(16.7 \%)\end{array}$ & $\begin{array}{c}43 \text { (91.5\%) } \\
4 \text { (8.5\%) }\end{array}$ & 0.232 \\
\hline $\begin{array}{l}\text { Smoking } \\
\text { Current or ex-smoker } \\
\text { Never smoker }\end{array}$ & $\begin{array}{c}43(89.6 \%) \\
5(10.4 \%)\end{array}$ & $\begin{array}{c}41(87.2 \%) \\
6(12.8 \%)\end{array}$ & 0.720 \\
\hline $\begin{array}{l}\text { ECOG PS } \\
0 \\
1\end{array}$ & $\begin{array}{c}1(2.1 \%) \\
47(97.9 \%)\end{array}$ & $\begin{array}{c}3(6.4 \%) \\
44(93.6 \%)\end{array}$ & 0.297 \\
\hline $\begin{array}{l}\text { First-line chemotherapy } \\
\text { Etoposide/cisplatin } \\
\text { Etoposide/carboplatin }\end{array}$ & $\begin{array}{l}31 \text { (64.6\%) } \\
17 \text { (35.4\%) }\end{array}$ & $\begin{array}{l}30 \text { (63.8\%) } \\
17(36.2 \%)\end{array}$ & 0.939 \\
\hline $\begin{array}{l}\text { Best response to etoposide/platinum } \\
\text { Complete response } \\
\text { Partial response } \\
\text { Stable disease }\end{array}$ & $\begin{array}{c}0 \\
44(93.6 \%) \\
3(6.4 \%)\end{array}$ & $\begin{array}{c}1(2.1 \%) \\
47(97.9 \%) \\
0\end{array}$ & 0.129 \\
\hline $\begin{array}{l}\text { Prophylactic cranial irradiation } \\
\text { Yes } \\
\text { No }\end{array}$ & $\begin{array}{c}5 \text { (10.4\%) } \\
43(89.6 \%)\end{array}$ & $\begin{array}{c}3 \text { (6.4\%) } \\
44 \text { (93.6\%) }\end{array}$ & 0.479 \\
\hline
\end{tabular}

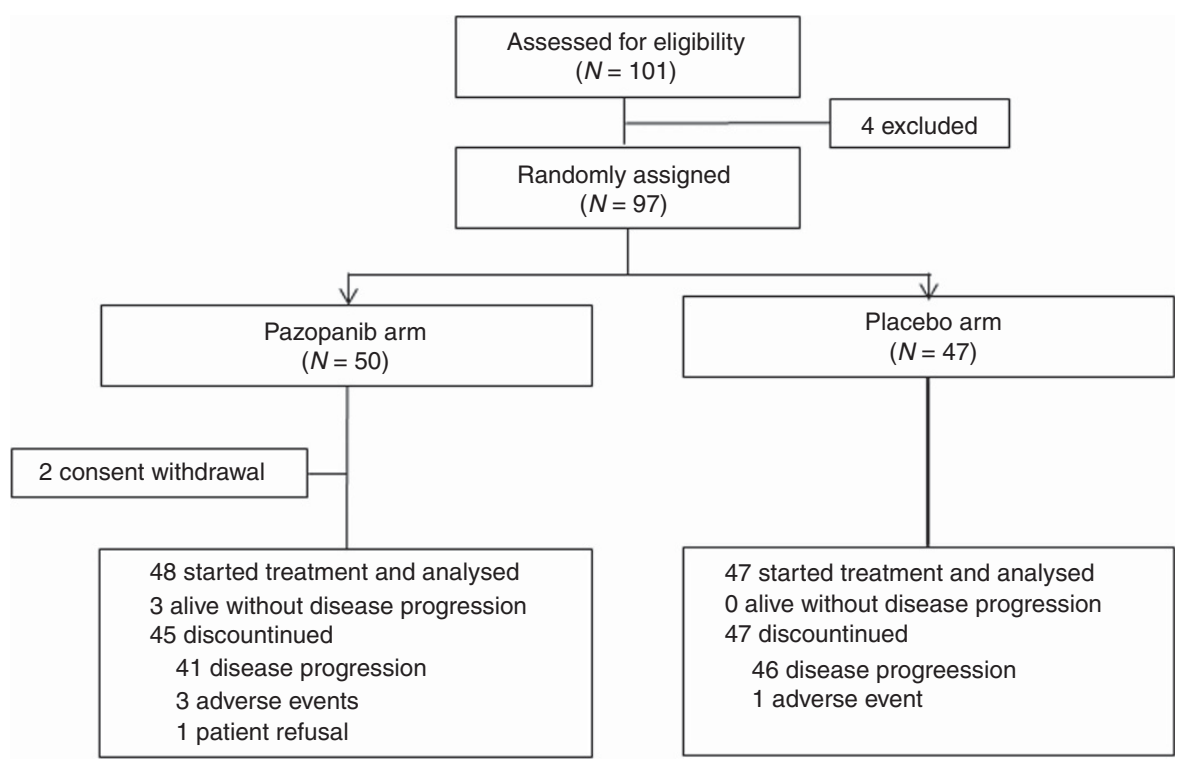

Figure 1. Trial profile. In total, 101 patients were assessed for eligibility and 97 were enroled and randomised. 95 were treated according to the study design and analysed by intention-to-treat analysis for efficacy and toxicity. 
Table 2. Adverse events

\begin{tabular}{|c|c|c|c|c|c|c|c|c|c|c|c|c|}
\hline & \multicolumn{6}{|c|}{ Pazopanib $(n=48)$} & \multicolumn{6}{|c|}{ Placebo $(n=47)$} \\
\hline & No. & $\%$ & No. & $\%$ & No. & $\%$ & No. & $\%$ & No. & $\%$ & No. & $\%$ \\
\hline AST elevation & 17 & 35.4 & 4 & 8.3 & 1 & 2.1 & 5 & 10.6 & 2 & 4.3 & 0 & 0.0 \\
\hline ALT elevation & 10 & 20.8 & 4 & 8.3 & 5 & 10.4 & 1 & 2.1 & 1 & 2.1 & 0 & 0.0 \\
\hline Skin lesions & 11 & 22.9 & 5 & 10.4 & 0 & 0.0 & 8 & 17.0 & 0 & 0.0 & 0 & 0.0 \\
\hline Diarrhoea & 8 & 16.7 & 6 & 12.5 & 1 & 2.1 & 2 & 4.3 & 0 & 0.0 & 0 & 0.0 \\
\hline Fatigue & 5 & 10.4 & 6 & 12.5 & 3 & 6.3 & 5 & 10.6 & 1 & 2.1 & 0 & 0.0 \\
\hline Stomatitis & 6 & 12.5 & 4 & 8.3 & 2 & 4.2 & 1 & 2.1 & 0 & 0.0 & 0 & 0.0 \\
\hline Myalgia & 0 & 0.0 & 2 & 4.2 & 1 & 2.1 & 1 & 2.1 & 0 & 0.0 & 0 & 0.0 \\
\hline
\end{tabular}

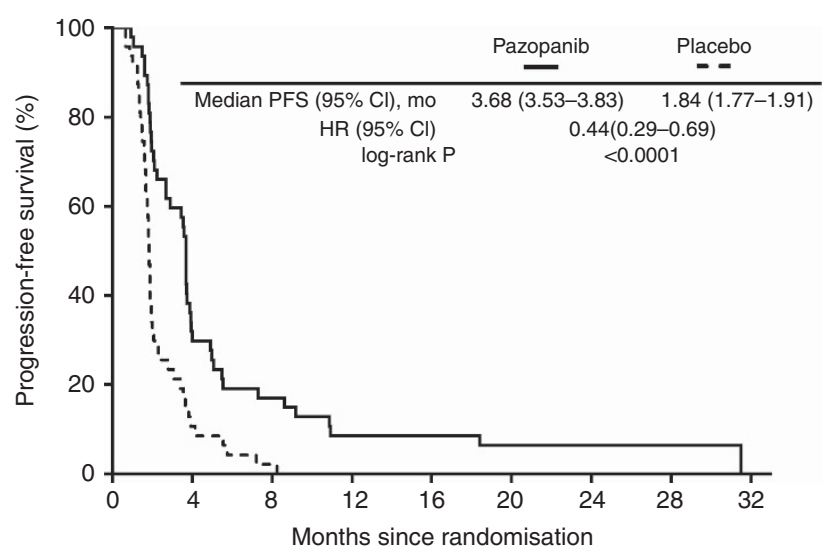

Figure 2. Progression-free survival. Pazopanib maintenance significantly improved PFS compared with placebo $(\mathrm{HR} 0.44,95 \% \mathrm{Cl}$ 0.29-0.69, $\mathrm{P}<0.0001) . \mathrm{Cl}=$ confidence interval; $\mathrm{HR}=$ hazard ratio; $\mathrm{PFS}=$ progression-free survival.

(95\% CI: 10.1-15.7 months), respectively (HR 1.14, 95\% CI: $0.74-$ $1.76, P<0.54$ ) (Figure 3$)$. In the unplanned exploratory analysis, there were no differences in overall survival according to history of PCI (PCI vs no PCI: 11.0 months vs 11.6 months, $P=0.40$ ) or regimen of first-line chemotherapy (etoposide plus cisplatin $v s$ carboplatin: 12.3 months $v s 9.8$ months, $P=0.64$ ).

Pattern of failure and post-study treatment. Out of 92 patients with documented disease progression during the study period, data on initial progression sites were available in 73 patients. Four (13.3\%) of 30 patients in the pazopanib arm and $6(14.0 \%)$ of 43 patients in the placebo arm experienced the brain as the first disease progression site $(P=0.94)$.

Out of 68 patients with available data about post-study treatment, $62(91.2 \%)$ received post-study chemotherapy. Twenty-seven $(84.4 \%)$ of 32 patients in the pazopanib arm received post-study chemotherapy, whereas $35(97.2 \%)$ of 36 patients in the placebo arm received subsequent chemotherapy $(P=0.062)$.The most common regimen as second-line chemotherapy was irinotecan-based

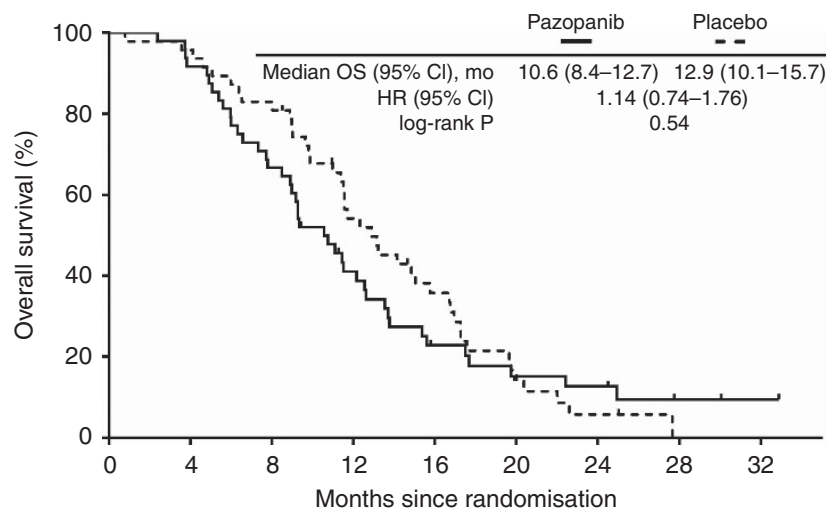

Figure 3. Overall survival. There was no difference in overall survival between the two arms (HR 1.14, 95\% Cl: 0.74-1.76, $P=0.54$ ). $\mathrm{Cl}=$ confidence interval; $\mathrm{HR}=$ hazard ratio; $\mathrm{OS}=$ overall survival.

chemotherapy $(n=57)$ and the other patients $(n=5)$ were treated again with etoposide plus platinum.

\section{DISCUSSION}

This study demonstrated a significant improvement of PFS, with a $56 \%$ reduction of risk ( $\mathrm{HR}, 0.44)$, for pazopanib maintenance in patients with ED-SCLC who had not progressed with first-line etoposide and platinum combination therapy. However, the PFS benefit has not translated into improved overall survival. The result of prolongation of PFS in the current study is consistent with previous studies using anti-angiogenic therapy in SCLC, which all demonstrated a significant improvement of PFS by addition of bevacizumab or sunitinib during or after first-line chemotherapy (Spigel et al, 2011; Ready et al, 2015; Tiseo et al, 2017). However, these studies failed to show an improvement in overall survival.

Given the significant improvement of PFS with pazopanib maintenance, the lack of a difference of overall survival between the two arms in this study might be affected by post-study treatment when crossover was not allowed. Although we did not 
prospectively collect data on post-study therapy, data were available for $74 \%$ of the patients with disease progression. Among them, all patients except six patients (five in the pazopanib and one in the placebo) did receive second-line chemotherapy. Therefore, we could assume that the prevalent use of second-line chemotherapy contributed in part to the decreased survival benefit from pazopanib maintenance in our study. Notably, however, all five patients who could not receive second-line therapy in the pazopanib arm died of cancer within 3 months after the studyoff. Among them, four patients experienced grade 2 adverse events during pazopanib maintenance. Although there was no available data explaining for the reason why they did not receive second-line therapy, their toxicity and dose reduction data implied that pazopanib maintenance therapy could affect negatively on their chance to receive second-line chemotherapy.

The observed safety profiles of pazopanib were quite similar to those of previous studies with pazopanib treatment in renal cell carcinoma, soft tissue sarcoma, and ovarian cancer (Sternberg et al, 2010; van der Graaf et al, 2012; Motzer et al, 2013; du Bois et al, 2014). However, the dose reduction rate (52\%) in the current study was slightly higher than in previous studies (39-44\%) (van der Graaf et al, 2012; Motzer et al, 2013). The higher dose reduction rates can in part be explained by that physicians tendency to reduce drug dose to maintain performance status and to control adverse events for long-term use of maintenance therapy, whereas these doses can otherwise be tolerated in active therapy for symptomatic metastatic disease. Another reason for the higher rate of dose reduction might be ethnic differences. In a pazopanib maintenance study for ovarian cancer, East Asians required more frequent dose reduction (75\%) than other participants (36\%), although the exact reason for this was not fully evaluated (du Bois et al, 2014). Nevertheless, compared with the sunitinib maintenance study in ED-SCLC (Ready et al, 2015), pazopanib maintenance showed more favourable toxicity profiles. In the CALGB 30504 trial, sunitinib maintenance with reduced dose $(37.5 \mathrm{mg})$ for ED-SCLC incurred higher incidences of grade 3 toxicities (total 53.5\%) and three patients had grade 4 adverse events (gastrointestinal hemorrhage; pancreatitis, hypocalcemia, and elevated lipase; and thrombocytopenia) (Ready et al, 2015).

In the current study, history of PCI was one of the stratification factors in the randomisation process to decrease potential biases; however, only $8 \%$ of participants received PCI before enrolment. As the protocol let physicians decide whether or not to perform PCI after completion of first-line chemotherapy, the use of PCI absolutely depended on the physicians' discretion. Currently, the role of PCI in ED-SCLC is controversial. The European Organisation for Research and Treatment of Cancer study reported that PCI improved overall survival $(P=0.003)$ and reduced the incidence of symptomatic brain metastases (Slotman et al, 2007). During the active enrollment of our study, however, a Japanese phase III study reported that PCI had detrimental effects on overall survival (10.1 months in the PCI arm vs 15.1 months in the placebo arm), although it reduced the risk of brain metastasis (32.2\% in the PCI arm vs 58.0\% in the placebo arm) (Takahashi et al, 2017). In addition, the neurotoxicity associated with PCI remains a concern and it has been reported that such neurotoxicity is more common in elderly patients (Wolfson et al, 2011; Gondi et al, 2013). Considering that the median age was 67 years in the current study, most of our patients might not be recommended for PCI by physicians.

It is of note that nine patients in the pazopanib arm experienced long-term PFS of more than 6 months, compared with only two patients in the placebo arm. Moreover, three patients in the pazopanib arm achieved additional partial response, whereas there was no objective response in the placebo arm. This indicates that there is a patient subpopulation that can benefit most from pazopanib maintenance therapy. To date, predictive biomarkers for anti-angiogenic agents in cancer therapy are still unclear, although a few studies provided several lines of evidence regarding potential biomarkers. High baseline plasma VEGF level was associated with better response to bevacizumab, but not survival, in non-small cell lung cancer (Dowlati et al, 2008). Baseline levels of cytokine and angiogenic factors such as hepatocyte growth factor and interleukin-12 were associated with tumour response to pazopanib in non-small cell lung cancer (Nikolinakos et al, 2010). As a biomarker study related to pazopanib was not performed in this study to explain the long-term PFS and additional partial response, relevant biomarkers to select patients that might benefit from pazopanib should be further investigated.

In summary, our study met the primary endpoint of PFS but failed to show an overall survival gain from pazopanib maintenance. Although a larger phase III study is needed to demonstrate the difference in overall survival based on our positive PFS results, we think further studies should be approached with different designs before we move forward, considering the current toxicity and overall survival data. One prerequisite for future studies can be an enrichment of study population based on predictive biomarkers that will most benefit from pazopanib maintenance therapy in terms of toxicity and efficacy.

\section{ACKNOWLEDGEMENTS}

We thank the patients who participated in the trial and the investigators who enroled patients for the study. The abstract of this study will be submitted to WCLC 2017 (Yokohama, Japan). This study was supported in part by Novartis. This study was supported by the Korea Health Technology R\&D Project through the Korea Heal Industry Development Institute, funded by the Ministry of Health \& Welfare, Republic of Korea (HI16C1984).

\section{CONFLICT OF INTEREST}

The authors declare no conflict of interest.

\section{REFERENCES}

Dowell JE, Amirkhan RH, Lai WS, Frawley WH, Minna JD (2004) Survival in small cell lung cancer is independent of tumor expression of VEGF and COX-2. Anticancer Res 24: 2367-2373.

Dowlati A, Gray R, Sandler AB, Schiller JH, Johnson DH (2008) Cell adhesion molecules, vascular endothelial growth factor, and basic fibroblast growth factor in patients with non-small cell lung cancer treated with chemotherapy with or without bevacizumab-an Eastern Cooperative Oncology Group Study. Clin Cancer Res 14: 1407-1412.

du Bois A, Floquet A, Kim JW, Rau J, del Campo JM, Friedlander M, Pignata S, Fujiwara K, Vergote I, Colombo N, Mirza MR, Monk BJ, Kimmig R, Ray-Coquard I, Zang R, Diaz-Padilla I, Baumann KH, Mouret-Reynier MA, Kim JH, Kurzeder C, Lesoin A, Vasey P, Marth C, Canzler U, Scambia G, Shimada M, Calvert P, Pujade-Lauraine E, Kim BG, Herzog TJ, Mitrica I, Schade-Brittinger C, Wang Q, Crescenzo R, Harter P (2014) Incorporation of pazopanib in maintenance therapy of ovarian cancer. J Clin Oncol 32: 3374-3382.

Eckardt JR, von Pawel J, Pujol JL, Papai Z, Quoix E, Ardizzoni A, Poulin R, Preston AJ, Dane G, Ross G (2007) Phase III study of oral compared with intravenous topotecan as second-line therapy in small-cell lung cancer. J Clin Oncol 25: 2086-2092.

Eisenhauer EA, Therasse P, Bogaerts J, Schwartz LH, Sargent D, Ford R, Dancey J, Arbuck S, Gwyther S, Mooney M, Rubinstein L, Shankar L, Dodd L, Kaplan R, Lacombe D, Verweij J (2009) New response evaluation criteria in solid tumours: Revised RECIST guideline (version 1.1). Eur J Cancer 45: 228-247. 
Gandhi L, Heist RS, Lucca JV, Temel JS, Fidias P, Morse LK, Johnson BE (2012) A phase II trial of pazopanib in relapsed/refractory small cell lung cancer (SCLC). J Clin Oncol 30: 7099.

Gondi V, Paulus R, Bruner DW, Meyers CA, Gore EM, Wolfson A, Werner-Wasik M, Sun AY, Choy H, Movsas B (2013) Decline in tested and self-reported cognitive functioning after prophylactic cranial irradiation for lung cancer: pooled secondary analysis of Radiation Therapy Oncology Group randomized trials 0212 and 0214. Int J Radiat Oncol Biol Phys 86: 656-664.

Govindan R, Page N, Morgensztern D, Read W, Tierney R, Vlahiotis A, Spitznagel EL, Piccirillo J (2006) Changing epidemiology of small-cell lung cancer in the United States over the last 30 years: analysis of the surveillance, epidemiologic, and end results database. J Clin Oncol 24: 4539-4544.

Hanna N, Bunn Jr PA, Langer C, Einhorn L, Guthrie Jr T, Beck T, Ansari R, Ellis P, Byrne M, Morrison M, Hariharan S, Wang B, Sandler A (2006) Randomized phase III trial comparing irinotecan/cisplatin with etoposide/ cisplatin in patients with previously untreated extensive-stage disease small-cell lung cancer. J Clin Oncol 24: 2038-2043.

Lucchi M, Mussi A, Fontanini G, Faviana P, Ribechini A, Angeletti CA (2002) Small cell lung carcinoma (SCLC): the angiogenic phenomenon. Eur $J$ Cardiothorac Surg 21: 1105-1110.

Motzer RJ, Hutson TE, Cella D, Reeves J, Hawkins R, Guo J, Nathan P, Staehler M, de Souza P, Merchan JR, Boleti E, Fife K, Jin J, Jones R, Uemura H, De Giorgi U, Harmenberg U, Wang J, Sternberg CN, Deen K, McCann L, Hackshaw MD, Crescenzo R, Pandite LN, Choueiri TK (2013) Pazopanib versus sunitinib in metastatic renal-cell carcinoma. $N$ Engl J Med 369: 722-731.

Nikolinakos PG, Altorki N, Yankelevitz D, Tran HT, Yan S, Rajagopalan D, Bordogna W, Ottesen LH, Heymach JV (2010) Plasma cytokine and angiogenic factor profiling identifies markers associated with tumor shrinkage in early-stage non-small cell lung cancer patients treated with pazopanib. Cancer Res 70: 2171-2179.

Ready NE, Pang HH, Gu L, Otterson GA, Thomas SP, Miller AA, Baggstrom M, Masters GA, Graziano SL, Crawford J, Bogart J, Vokes EE (2015)

Chemotherapy with or without maintenance sunitinib for untreated extensivestage small-cell lung cancer: a randomized, double-blind, placebo-controlled phase II study-CALGB 30504 (Alliance). J Clin Oncol 33: 1660-1665.

Schiller JH, Adak S, Cella D, DeVore 3rd RF, Johnson DH (2001) Topotecan versus observation after cisplatin plus etoposide in extensive-stage small-cell lung cancer: E7593-a phase III trial of the Eastern Cooperative Oncology Group. J Clin Oncol 19: 2114-2122.

Slotman B, Faivre-Finn C, Kramer G, Rankin E, Snee M, Hatton M, Postmus P, Collette L, Musat E, Senan S. Eortc Radiation Oncology Group, Lung Cancer Group (2007) Prophylactic cranial irradiation in extensive small-cell lung cancer. N Engl J Med 357: 664-672.
Sonpavde G, Hutson TE (2007) Pazopanib: a novel multitargeted tyrosine kinase inhibitor. Curr Oncol Rep 9: 115-119.

Spigel DR, Townley PM, Waterhouse DM, Fang L, Adiguzel I, Huang JE, Karlin DA, Faoro L, Scappaticci FA, Socinski MA (2011) Randomized phase II study of bevacizumab in combination with chemotherapy in previously untreated extensive-stage small-cell lung cancer: results from the SALUTE trial. J Clin Oncol 29: 2215-2222.

Sternberg CN, Davis ID, Mardiak J, Szczylik C, Lee E, Wagstaff J, Barrios CH, Salman P, Gladkov OA, Kavina A, Zarba JJ, Chen M, McCann L, Pandite L, Roychowdhury DF, Hawkins RE (2010) Pazopanib in locally advanced or metastatic renal cell carcinoma: results of a randomized phase III trial. J Clin Oncol 28: 1061-1068.

Takahashi T, Yamanaka T, Seto T, Harada H, Nokihara H, Saka H, Nishio M, Kaneda H, Takayama K, Ishimoto O, Takeda K, Yoshioka H, Tachihara M, Sakai H, Goto K, Yamamoto N (2017) Prophylactic cranial irradiation versus observation in patients with extensive-disease small-cell lung cancer: a multicentre, randomised, open-label, phase 3 trial. Lancet Oncol 18: 663-671.

Tiseo M, Boni L, Ambrosio F, Camerini A, Baldini E, Cinieri S, Brighenti M, Zanelli F, Defraia E, Chiari R, Dazzi C, Tibaldi C, Turolla GM, D'Alessandro V, Zilembo N, Trolese AR, Grossi F, Riccardi F, Ardizzoni A (2017) Italian, multicenter, phase III, Randomized study of cisplatin plus etoposide with or without bevacizumab as first-line treatment in extensive-disease small-cell lung cancer: the GOIRC-AIFA FARM6PMFJM trial. J Clin Oncol 35: 1281-1287.

van der Graaf WT, Blay JY, Chawla SP, Kim DW, Bui-Nguyen B, Casali PG, Schoffski P, Aglietta M, Staddon AP, Beppu Y, Le Cesne A, Gelderblom H, Judson IR, Araki N, Ouali M, Marreaud S, Hodge R, Dewji MR, Coens C, Demetri GD, Fletcher CD, Dei Tos AP, Hohenberger P (2012) Pazopanib for metastatic soft-tissue sarcoma (PALETTE): a randomised, double-blind, placebo-controlled phase 3 trial. Lancet 379: 1879-1886.

Wolfson AH, Bae K, Komaki R, Meyers C, Movsas B, Le Pechoux C, Werner-Wasik M, Videtic GM, Garces YI, Choy H (2011) Primary analysis of a phase II randomized trial Radiation Therapy Oncology Group (RTOG) 0212: impact of different total doses and schedules of prophylactic cranial irradiation on chronic neurotoxicity and quality of life for patients with limited-disease small-cell lung cancer. Int J Radiat Oncol Biol Phys 81: 77-84.

This work is published under the standard license to publish agreement. After 12 months the work will become freely available and the license terms will switch to a Creative Commons AttributionNonCommercial-Share Alike 4.0 Unported License. 